\title{
Prevalence of Epstein-Barr Virus Latent Membrane Protein-1 in Jordanian Patients with Hodgkin's Lymphoma and Non- Hodgkin's Lymphoma
}

Fawzi Irshaid, Adnan Jaran, Fatiha Dilmi, Khaled Tarawneh, Raji Hadeth, and Ahad Al-Khatib

\begin{abstract}
The aim of this study was to estimate the frequency of EBV infection in Hodgkin's lymphoma (HL) and non-Hodgkin's lymphoma (NHL) occurring in Jordanian patients. A total of 55 patients with lymphoma were examined in this study. Of 55 patients, 30 and 25 were diagnosed as HL and NHL, respectively. The four HL subtypes were observed with the majority of the cases exhibited the mixed cellularity (MC) subtype followed by the nodular sclerosis (NS). The high grade was found to be the commonest subtype of NHL in our sample, followed by the low grade. The presence of EBV virus was detected by immunostating for expression of latent membrane protein-1 (LMP-1). The frequency of LMP-1 expression occurred more frequent in patients with HL $(60.0 \%)$ than in patients with NHL (32.0\%). The frequency of LMP-1 expression was also higher in patients with MC subtype (61.11\%) than those patients with NS $(28.57 \%)$. No age or gender difference in occurrence of EBV infection was observed among patient with HL. By contrast, the prevalence of EBV infection in NHL patients aged below 50 was lower $(16.66 \%)$ than in NHL patients aged 50 or above (46.15\%). In addition, EBV infection was more frequent in females with NHL $(38.46 \%)$ than in male with NHL $(25 \%)$. In NHL cases, the frequency of EBV infection in intermediate grade $(60.0 \%)$ was high when compared with frequency of low $(25 \%)$ or high grades $(25 \%)$. In conclusion, analysis of LMP-1 expression indicates an important role for this viral oncogene in the pathogenesis of EBV-associated malignant lymphomas. These data also support the previous findings that people with EBV may develop lymphoma and that efforts to maintain low lymphoma should be considered for people with EBV infection.
\end{abstract}

Keywords-Hodgkin lymphoma, Epstein Barr virus, hematoxylin, infection, LMP-1 expression.

\section{INTRODUCTION}

$\mathrm{L}$ YMPHOMA is broadly divided into Hodgkin's lymphoma (HL) and Non-Hodgkin's Lymphoma (NHL) HISbased on classification of the World Health Organization [1]. HL is also subclassified into two four categories termed nodular sclerosis (NS), mixed cellularity (MC), lymphocyte depletion (LD), and lymphocyte-rich (LR). In addition, the Working Formulation Classification has been adopted in United States. According to this system NHL has been divided

Correspondence: Dr. Fawzi Irshaid is with Department of Biological Sciences, Faculty of Science, Al al-Bayt University, P. O. Box 130040, AlMafraq 25113, Jordan. Office: 962-2-6297000, Telfax: 0096265151261, email: irshaid@aabu.edu.jo. into low, intermediate and high grade. In Europe and elsewhere, the Kiel classification of NHL has been accepted widely. Based on the Kiel classification, NHL is divided into B-cell and T-cell lineages based on available immunophenotypic data and into low or high grades based on cell morphology.

According to data from the Jordanian National Cancer Registry in 2004, the estimated number of newly diagnosed cancer cases was approximately 5000 cases per year. This estimate may be less far from the accurate number [2]. This is because cancer database is generally incomplete or inaccurate and several numbers of cases were officially not recognized or reported. Importantly, because cancer among these patients is commonly discovered in advance stage, about $70 \%$ of these patients will die of cancer within the first year of their diagnosis. In addition, approximately $5.7 \%$ of these cancer cases were diagnosed with lymphoma. Moreover, data from The International Agency for Research on cancer indicated that at least 287,000 of NHL and 62,000 of HL new cases were annually diagnosed world wide. In USA, NHL is already ranked fifth among white male cancers for both incidence and mortality and sixth for female cancers [3]. In addition, the prevalence of NHL has increased over the past two decades, with median age of 55-60 years at presentation and the incidence increases with advancing age. Recently, there was some evidence of complete cure of lymphoma by irradiation and chemotherapy. The best results were achieved for those patients when intensifying radiotherapy and chemotherapy were administered earlier rather than later in the course of the disease [4], [5]. However, it has been become clear that death due to second cancers are now the most common cause of mortality among long-term survivors of HL, followed by cardiac disease [6], [7].

The search for a cause of cancers has raveled that the Epstein Barr Virus (EBV) appears to be associated with some cancers including lymphoma [8], [9]. EBV is a very common virus that is already infecting high percent of population worldwide and persisting for the lifetime in the host [10]. Importantly, several studies indicated that EBV is linked to the development of multiple cancers, including Burkitt's lymphoma, HL, NHL and some diffuse large B-cell lymphomas [8], [9]. Despite the fact that EBV is a common infection, it has been postulated that EBV play a role in the 
pathogenesis of lymphoma [8]. The association between lymphoma and EBV came from the observation that EBV is the causative agent of infectious mononucleosis [8]-[11]. So that patients with a previous history of infectious mononucleosis have an elevated risk of developing lymphoma.

Furthermore, a reasonable interpretation for the association of EBV with Hodgkin's disease is that EBV can transform resting human B-cells into permanently proliferating lymphoblastoid cell lines that constitutively expresses LMP-1 [10]-[14]. Transcription of this viral oncogene was demonstrated in various malignant lymphoma cells. In addition, it has been proposed that the expression of LMP-1 might provide growth and survival signals that enhance tumor growth and hence contribute to the malignant phenotype of Bcells [10]-[15]. It is also important to mention that a germinalcenter $\mathrm{B}$ cell might acquire a mutation during infectious mononucleosis that blocks its differentiation [11]-[15]. Thus, we anticipate that an earlier detection and treatment of EBV infection most likely would contribute to decrease the rate of lymphoma incidence among our population. Moreover, little is known about the risk of EBV infection for the substantial majority of lymphoma patients in Jordan. In this study our goal, therefore, was to estimate the prevalence of EBV infection in Jordanian patients diagnosed with HL and NHL using immunohistochemistry technique with primary monoclonal antibody against EBV-LMP-1.

\section{MATERIALS AND METHODS}

\section{A. Samples collection}

A total of 55 samples diagnosed with malignant lymphoma were randomly selected from the archive of pathology laboratory of King Hussein Hospital, Amman, Jordan. The samples were available as paraffin embedded tissues. All lymphoma tissues were examined microscopically, and then divided into HL and NHL. These HL and NHL samples were further subdivided into different grades or subtypes by Dr. Raji Hadeth, a pathologist from faculty of Medicine, Jordan University of Sciences and Technology, Irbid, Jordan.

\section{B. Hematoxylin and Eosin (H \& E) Staining}

Formalin-fixed paraffin tissues were sectioned at $4 \mu \mathrm{m}$ thick. These sections were deparaffinized by heating and then immediately cleared in xylene. This was followed by rehydration through serial dilutions of alcohol. These sections were counterstained with hematoxylin and stained with eosin before dehydration. Finally, these sections were mounted with distrenedibutylphthlate-xylene (DPX) and examined under light microscope (Nikon, Japan).

\section{Immunohistochemistry study}

Immunohistochemical staining was done on $4 \mu \mathrm{m}$ thick consecutive sections of formalin fixed, paraffin-embedded tissue specimens. Tissue section slides were deparaffinized and rehydrated as described above. Then, slides were immersed in 3\% hydrogen peroxidase in methanol for 10 minutes to block endogenous peroxidase activity and rinsed in running water. Subsequently slides were treated under pressure in the commercial solution (Reveal 10X, Biocare Medical, CA, USA) in the Decloaking chamber (Biocare Medical) to retrieve the antigens and block endogenous biotin. Then, slides were allowed to cool at room temperature for $20 \mathrm{~min}$. This was followed by washing and incubation with phosphate buffer saline (PBS) (diluted at 1:10) for 15 min. Slides were then incubated with primary mouse monoclonal antibody against EBV-LMP-1 for 30 min (diluted at 1:50, from Biocare medical). One drop of biotinylated goat anti-mouse (diluted at 1:10; from BioGenex, San Ramon, CA, USA) was added to each slide, and washed well with PBS. After that, slides were incubated with streptavidin peroxidase (diluted at 1:10, from BioGenex) for $20 \mathrm{~min}$ at room temperature and washed well with PBS. Slides were then incubated with 3,3-Diaminobenzidine chromogen (from BioGenex). This was followed by counterstained with hematoxylin and then stained with eosin before dehydration and mounted with DPX. Finally, all slides were examined under light microscope (Nikon, Japan). A previously wellknown negative case for EBV was used as negative control and treated as described above.

\section{Immunostaining assessment and evaluation}

In NHL specimens with less than 5\% EBV-positive tumor cells were judged as negative and specimens with more than $5 \% \mathrm{EBV}$-positive tumor cells were interpreted as positive. It is worth to mention that the percentage of EBV-positive staining whether membrane or paranuclear were taken into consideration. Whereas in HL, the specimen is considered EBV-positive if any Reed-Sternberg (RS) cell was membrane or paranuclear staining positive. This is because the HL is usually characterized by the scarcity of the neoplastic RS cell $(1 \%-10 \%)$.

\section{Results}

In the present study, we examined 55 samples which fulfilled the lymphoma criteria. There were 30 patients HL and 25 patients were NHL. Male patients with HL were 20

$(66.7 \%)$ while $10(33.3 \%)$ were female patients with HL. The male versus female ration was $2: 1$. Of $30 \mathrm{HL}$ cases, 24 $(80 \%)$ were below the age of 50 years while six out of the 30 cases $(20 \%)$ occurred in age 50 years or above (Table I). As can be seen in Fig. 1, the age group distribution of our HL cases was

as follows: 0-9 years 1 (3.3\%), 10-19 years $10(33.3 \%)$, where the highest incidence occurred, $20-29$ years $5(16.7 \%)$, $30-39$ years $4(13.3 \%), 40-49$ years $4(13.3 \%), 50-59$ years 6 (19.9), 60-69 years 4 (13.3\%), and 70 years and above 1 (3.3\%). The median age was 28 years and ranged from 4-70 years. On other hand, there were 12 males (48\%) and 13 female $(52 \%)$ with NHL. The male versus female ration was 1:0.92. Of 25 NHL cases, 12 (48\%) were below the age of 50 years and $13(52 \%)$ were at the age of 50 years and above. The age group distribution of our NHL cases was as follows (Fig. 1): $0-9$ years $4(16 \%), 10-19$ years $3(12 \%), 20-29$ years 
$1(4 \%), 30-39$ years $1(4 \%), 40-49$ years $3(12 \%), 50-59$ years $3(12 \%), 60-69$ years $4(16 \%)$, and 70 years and above 6 (24\%). Unlike HL, no peak of incidences in NHL was noted among the different age groups. The median age was 50 years and ranged from 3-78 years.

The HL samples were further divided into subgroups according to the WHO classification criteria. HL cases were divided into 5 subtypes. Based on this classification, there were $60 \%(18 / 30)$ mixed cellularity (MC), 23.33\% (7/30) nodular sclerosis (NS), 6.66\% (2/30) lymphocyte depletion (LD), 4.33\% (1/30) lymphocyte rich (LR) and 6.66\% (2/30) unclassified (UC), as shown in Table II. On other hand, NHL was graded into high, intermediate and low grade according to the International Working Formulation system. There were $48 \%(12 / 25)$ of high grade, $20 \%(5 / 25)$ of intermediate and $32 \%(8 / 25)$ of low grade (Table II). The presence of EBV was confirmed by the expression of LMP-1 using specific monoclonal antibodies. LMP-1 usually displays a typical membrane staining in tumor cells. In this work, staining results of the investigated LMP-1 marker were used to classify the HL and NHL samples into positive only when tumor cells stained with the corresponding marker and thus, the absence of such staining pattern was considered negative as shown in Fig. 2 and 3. Overall, the high incidence of EBV-LMP-1 protein observed in HL cases $60 \%(18 / 30)$, in contrast to the relatively low incidence observed in NHL cases $32 \%(8 / 25)$. Our immunohistochemical staining results also indicated that the EBV-LMP-1 protein expression was observed in 15 of 24 $(62.50 \%)$ HL cases with below 50 years of age, and a closely similar frequency was observed in 3 of $6(50.00 \%)$ HL cases with 50 years of age and above (Table III). In addition, a similar frequency of EBV-LMP-1 expression was observed in both male $60.00 \%(12 / 20)$ and female $60.00 \%(6 / 10)$ with HL, as shown in Table III. With regards to subtype (Table IV), the presence of EBV-LMP-1 was detected in 11 of $18(61.11 \%)$ MC cases and 2 of 7 (28.57\%) NS cases. On other hand, expression of LMP-1 of EBV was observed in all cases of other subtypes, including LD, LR and UC.

Furthermore, with respect to gender patients with NHL, the presence of EBV was detected in 3 of $12(25 \%)$ male cases and 5 of $13(38.46 \%)$ female cases (Table III). Our data also revealed that EBV-LMP-1 was found in 2 of $12(16.66 \%)$ NHL cases with below 50 years of age, in contrast to the relatively high incidence observed in 6 of $13(46.15 \%)$ NHL cases with 50 years of age and above. With regard to grade, the presence of EBV was detected in 2 of $8(25.00 \%)$ low grade cases, 3 of $5(60.0 \%)$ intermediate grade cases and 3 of $12(25.00 \%)$ high grade cases (Table IV).

\section{DISCUSSION}

Our current study indicated that the HL accounted 55\% and $45 \%$ for NHL. Previously, it was reported that NHL accounted for as high as $60.7 \%$ and $78.4 \%$ of all lymphoma cases among the Jordanian population [16]. Additionally, different studies from the Middle East have also reported higher prevalence of NHL compared to HL, namely Oman with $65 \%$ NHL and 35\% HL [17], Bahrain with $66.7 \%$ NHL and $33.3 \%$ HL [18] and United Emirates with 59\% NHL, and $41 \%$ HL [19]. This finding might be explained by the nature of our samples. In fact, our samples were randomly selected from 300 available samples, of which 190 samples were found in well preserved and adequate amount that might explain the high incidence of HL. It is also important to note that some of lymphomas previously diagnosed as HL are now classified as NHL and this shift is responsible for the declining incidence of HL.

Available data reported that lymphoma can occur in all age group [8], [9], [16], [17]. Our data indicated that a clear difference was observed in the incidence of HL among different age groups with the majority of cases $(80 \%)$ occurring in the younger age groups below 50 years versus $20 \%$ of cases occurring above or equal that age. Similar findings were also reported in certain areas such as Cyprus with $84.3 \%$ of HL below 50 years and Egypt with $84.4 \%$ below 50 years [20]. In addition, 33.3\% of the HL cases fell within the age interval 10 to 19 years which represented an early major peak of incidence. An observation in this study correlates very well with the previous research findings, in that HL age specific incidence with one peak between 11 and 20 years was reported in other studies [16], [21]. The slightly difference between these study and our study are more likely due to small size of our sample. Moreover, it has been reported that the overall incidence of HL has decreased in parallel with a slight increase in young adults during the past decades worldwide. Unlike HL, 52\% of NHL cases fill above age of 50 years, and no peak of incidence was noted among NHL cases at different age groups. This result is in agreement with the results of study in Egypt with $49.5 \%$ NHL cases below 50 years [20]. Our and these data clearly suggest that NHL cases seem to be distributed somewhat regularly within different age groups. By contrast, HL seems to occur more frequently in male than in female, (with a male to female ratio: $2: 1)$. The overall frequency of HL in male and female is entirely consistent with the published literature, minor differences almost certainly reflecting differences in sample size. However, no sex difference was observed in NHL where the sex ratio was approximately equal.

Among the various subtypes of HL, the MC was found to be the commonest subtype in our study $(60 \%)$ followed by the NS (23.3\%). Our results are similar to recent estimate from Iraq with predominance of MC $(67.5 \%)$ followed by NS $(12 \%)$ [22]. However, these results are in contrast with western countries where the NS is the most frequent subtype. For example, in Italy and USA, NS was estimated to be about $75 \%-80 \%$ of all HL cases [23]. Moreover, the most frequent grades of NHL were the high grade $(48 \%)$, followed by low grade $(32 \%)$. This result differed from previous study in Saudi Arabia which reported that low, intermediate and high grade of NHL constituted about $18 \%, 37 \%$ and $29 \%$, respectively [24]. These unexpected findings, however, should be taken cautiously in that it difficult to explain. In addition, these minor differences may be a coincident or required another 
study with larger sample size. Taken together, the variations in the prevalence of NHL in various studies could be due to different study methodologies of classifications, difference in sample sizes and/or misclassification due to the unspecialized personnel used to classify lymphoma. It could also be due to some environmental factors or other unknown causes.

It has been proposed that LMP-1 is expressed in many EBV-associated cancers and is responsible for most of the altered cellular growth properties that are induced by EBV infection. In this work, staining results of the LMP-1 were used to sub-classify the NH and NHL into positive EBV or negative EBV. Our staining data revealed that the frequency of expression of LMP-1 of EBV was higher in HL patients $(60 \%)$ than in NHL patients $(32 \%)$. These data are in agreement with the above data which revealed that the HL occurred more frequent than NHL in Jordanian patients with lymphoma. Also, our current result correlates very well with the previous research findings [22], [25]. These observations further support the notion that expression of LMP-1 of EBV might involved in development and progression of HL. However, other study showed that incidence of EBV in developed country was $50 \%$ in their HL cases [8]-[12], whereas others reported that EBV expression was more than $90 \%$ in underdeveloped countries [14], [22]-[25]. Taken together, these findings also suggest that efforts to maintain low lymphomas must be considered for people with EBV infection.

With regard to histological subtypes and their association with EBV, our findings indicated that EBV expression was higher in the MC (61.11\%), followed by NS (28.57\%). These results were in consistent with results in previous study which showed that the EBV expression was higher in MC (66.6\%), followed by NS (46.1\%) [25]. It is also worth to note that all cases of the LD, LP and UC subtypes were found to be positive for EBV. The high prevalence of EBV in these subtypes should be interpreted cautiously due to the small number of cases in each category.

The presence of EBV seems to occur more frequent in HL $(60.0 \%)$ than NHL cases $(32.0 \%)$. These results were in agreement with some previously studies which also found $30 \%$ EBV positive in their NHL cases [22], [26]. On other hand, different studies reported higher association of the EBV with NHL as high as $80 \%$ in other developing countries [27], [10]. It is also worth mentioning that Burkitt's lymphoma is a high-grade malignant NHL that is most commonly associated with EBV infection [10], [12], [22], [27]. Furthermore, in Africa, Burkitt's lymphoma is associated with Plasmodium falciparum malaria, and tumors usually present in the jaw, over 90 percent of these cases are associated with EBV. In the United States, patients with Burkitt's lymphoma usually present with abdominal tumors, only 20 percent of which are associated with EBV [10]. Therefore, the low rate of LMP-1 of EBV positive in NHL cases that has been seen in our study may be due to little cases of Burkitt's lymphoma in our sample. This was clearly evident from our study which reported only one case in our sample. It is important to mention that the mode of transmission in our population is still uncertain. Therefore, it is worth to investigate the mode of transmission of EBV in our population with larger sample sizes of lymphoma patients.

Furthermore, the frequency of EBV in intermediate grade was much higher with approximately $60 \%$ of cases with EBV positive compared to the high or low grade cases, where about $25 \%$ were EBV positive. The overall frequency of expression of LMP-1 of EBV in these different grades is somewhat consistent with the published literature with minor differences. Taken together, the above studies confirm that the association of EBV with lymphoma is strongly but variably linked to various environmental factors prevailing in different geographic regions. These include the economic status, geographical location, ethnic variations, and health conditions of studied areas as well as the great diversity of lymphoma subtypes.

\section{CONCLUSION}

The data derived from our lymphoma sample indicated for the first time that the percentage of HL cases was slightly higher than that of NHL cases. Similarly, frequency of expression of LMP-1 of EBV was higher in HL patients than in NHL patients. The data also showed that the age, sex and clinical characteristics of Jordanian patients with HL and NHL included in this study are slightly different from those of neighbor countries. Furthermore, we suggest that a high sensitive technique must be carried out to verifying our observations.

The present study also provided evidence supporting the previous findings that people with EBV might develop lymphoma. Thus, there is an urgent need to educate our people at different age about the potential threat that EBV infection might cause to them. These findings also provided an additional reason for doctors to take EBV infection very seriously in patients with lymphomas. In addition, our results should encourage physicians to achieve complete viral suppression treatment for those patients with persisting EBV infection in an attempt to minimize the risk of this lifethreatening disease.

\section{ACKNOWLEDGMENT}

This study has been supported in part by the Deanship of Scientific Research at Al al-Bayt University. The financial and technical supports from Al al-Bayt University are gratefully acknowledged. We would also like to thanks Dr. Ali chouter, medical staff in forensic laboratory in Irbid and medical research staff in Jordan University of Science and Technology for their valuable advice, technical support and assistance.

\section{REFERENCES}

[1] N. L. Harris, E. S. Jaffe and J. O. Armitage, et al., "Lymphoma classification: from R.E.A.L. to W.H.O. and beyond," Cancer: Principles and Practice of Oncology Updates, vol. 13, pp. 1-14, 1999.

[2] J. Stjernswärd, F. Ferris, S. Khleif, W. Jamous, I. Treish, M. Milhem, M. Bushnaq, A. Al Khateib, N. Al-Shtiat, M. Wheeler, and A. Alwan, 
"Jordan palliative care initiative: A WHO demonstration project," Journal of Pain and Symptom Management, vol. 33, pp. 628-633, 2007.

[3] A. Jemal, A. Thomas, T. Murray, and M. Thun, "Cancer statistics CA," Cancer J. Clin. Vol. 52, pp. 23-47, 2002.

[4] J. G. Gribben, "How I treat indolent lymphoma," Blood, vol. 109, pp 4617-4626, 2002.

[5] L. B. Travis, "Evaluation of the risk of therapy-associated complications in survivors of Hodgkin Lymphoma," Hematology, vol. 1, pp. 192-196, 2007.

[6] J. S. Knight, A. Tsodikov, D. M. Cibrik, C. W. Ross, M. S. Kaminski, and D. W. Blayney, "Lymphoma after solid organ transplantation: Risk, response to therapy, and survival at a transplantation center," J. Clin. Oncol., vol. 27, pp. 3354-3362, 2009.

[7] K. Hemminki, P. Lenner, J. Sundquist, and J. L. Bermejo, "Risk of subsequent solid tumors after non-Hodgkin's lymphoma: Effect of diagnostic age and time since diagnosis," J. Clin. Oncol., vol. 26, pp. 1850-1857, 2008.

[8] S. B. Pattle, and P. J. Farrell, "The role of Epstein-Barr virus in cancer," Expert Opin. Biol. Ther., Vol. 6, pp. 1193-1205, 2006.

[9] D. Serraino, P. Piselli, C. Angeletti, M. Scuderi, and M. R. Ippolito, "Infection with Epstein-Barr virus and cancer: an epidemiological review," J. Biol. Regul. Homeost. Agents, vol. 19, pp. 63-70, 2005.

[10] J. I. Cohen, "Epstein-Barr virus infection," N. Engl. J. Med., vol. 343, pp. 481-492, 2000.

[11] G. Kapatai, and P. Murray, "Contribution of the Epstein Barr virus to the molecular pathogenesis of Hodgkin lymphoma," J. Clin. Pathol., vol. 60, pp. 1342-1349, 2007.

[12] D. A. Thorley-Lawson, and A. Gross, "Persistence of the Epstein-Barr virus and the origins of associated lymphomas," N. E. J. M., vol. 350, pp. 1328-1337, 2004.

[13] L. H. Kim, S. C. Peh, and S. Poppema, "Expression of retinoblastoma protein and p16 proteins in classic Hodgkin's lymphoma: relationship with expression of p53 and presence of Epstein-Barr virus in the regulation of cell growth and death," Journal of Human Pathology, vol. 37, pp. 92-100, 2006.

[14] P. Zhao, Y. Lu, L. Liu, and M. Zhong, "Aberrant expression of ID2 protein and its correlation with EBV-LMP1 and p16 (INK4A) in Classical Hodgkin's Lymphoma in China," BioMed Central journal, vol. 8, pp. 1471-2407, 2008.

[15] L. M. Staudt, "The molecular and cellular origins of Hodgkin's disease," The Journal of Experimental Medicine, vol. 191, pp. 207-212, 2002.

[16] W. J. Haddadin, "Malignant lymphoma in Jordan: a retrospective analysis of 347 cases according to the World Health Organization classification," Annals of Saudi Medicine, vol. 25, pp. 398-403, 2005.

[17] S. Bamanikar, S. Thunold, K. R. Devi, and A. Bamanikar, "The pattern of malignant lymphoma in Oman," Tropical Medicine and Hygiene Journal, vol. 98, pp. 351-354, 1995.

[18] D. K. Shome, S. M. George, F. Al-Hilli, and A. A. Satir, "Spectrum of malignant lymphomas in Bahrain. Leitmotif of a regional pattern," Saudi Medicine Journal, vol. 25, pp. 146-167, 2004.

[19] A. Castella, S. Joshi, T. Raaschou, and N. Mason, "Pattern of Malignant Lymphoma in the United Arab Emirates," Acta Oncologica, vol. 40, pp. 660-664, 2001.

[20] A. S. Soliman, and P. Boffetta, "lymphoma and leukemia," Middle East Cancer Consortium (MECC) Registries, 2004.

[21] N. M. Almasri, "Hodgkin's lymphoma in north Jordan. Does it have different pattern," Saudi Medical Journal, vol. 25, pp. 1971-1921, 2004.

[22] W. K. Ridha, "Epstein Barr Virus-Mediated deregulation of cell cycle pathways in malignant lymphomas: implication of NFKB, p53, p27 and the mutant p21 Ras using in situ technique," Ph. D. Thesis, University of AL-Nahrain, Iraq, 2004.

[23] S. A. Pileri, S. Ascani, L. Leoncini, E. Sabattini, P. L. Zinzani, P. P. Piccaluga, A. Pileri, M. Giunti, B. Falini, G. B. Bolis, and H. Stein, "Hodgkin's lymphoma: the pathologist's viewpoint," Journal of Clinical, vol. 55, pp. 162-176, 2002.

[24] J. O. Thomas, and M. A. Abdullah, "Malignant lymphoma in western province of Saudi Arabia," East African Medical Journal, vol. 72, pp. $355-358,1995$.

[25] J. Vassallo, K. Metze, F. Traina, C. de Souza, and I. Lorand-Metze, "Expression of Epstein-Barr virus in classical Hodgkin's lymphomas in Brazilian adult patients," Haematologica, vol. 86, pp.1227-1228, 2001.

[26] S. Bossolasco, P. Cinque, M. Ponzoni, M. G. Vigano, A. Lazzarin, A Linde, and K. I. Falk, "Epstein-Barr virus DNA load in cerebrospinal fluid and plasma of patients with AIDS-related lymphoma," Journal of NeuroVirology, vol. 8, pp. 432- 438, 2002.

[27] C. R. Rao, M. I. Gutierrez, K. Bhatia, F. Fend, J. Franklin, L. Appaji, G. Gallo, G. Oconor, N. Lalitha, and I. Magrath, "Association of Burkitt's lymphoma with the Epstein-Barr virus in two developing countries," Leukemia and Lymphoma, vol. 39, pp. 329-337, 2002. 
World Academy of Science, Engineering and Technology

International Journal of Biotechnology and Bioengineering Vol:4, No:12, 2010

TABLE I

DISTRIBUTION OF HODGKIN'S LYMPHOMA (HL) AND NON-HODGKIN'S LYMPHOMA (NHL) PATIENTS ACCORDING TO GENDER AND AGE AT DIAGNOSIS

\begin{tabular}{c|cccc} 
Type of lymphoma & \multicolumn{2}{|c}{ Age groups } & \multicolumn{2}{c}{ Gender } \\
$<50$ yrs & $\mathrm{N}(\%)$ & $\begin{array}{l}\text { Male yrs } \\
\mathrm{N}(\%)\end{array}$ & $\mathrm{N}(\%)$ & $\begin{array}{l}\text { Female } \\
\mathrm{N}(\%)\end{array}$ \\
\hline $\mathrm{HL}$ & $24(80.0)$ & $6(20.0)$ & $20(66.6)$ & $10(33.3)$ \\
$\mathrm{NHL}$ & $12(48.0)$ & $13(52.0)$ & $12(48.0)$ & $13(52.0)$ \\
\hline
\end{tabular}

TABLE II

DISTRIBUTION OF HODGKIN'S LYMPHOMA (HL) AND NON-HODGKIN'S LYMPHOMA (NHL) PATIENTS ACCORDING TO SUBTYPE AND GRADE.

\begin{tabular}{c|lcc}
\hline \multirow{2}{*}{ Type of lymphoma } & \multicolumn{1}{|c}{ Subtype } & R & \\
& & & \\
& & 18 & 60.00 \\
& Mixed Cellularity & 7 & 23.33 \\
& Nodular Sclerosis & 2 & 6.66 \\
& Lymphocyte Depletion & 1 & 4.33 \\
& Lymphocyte Rich & 2 & 6.66 \\
& Unclassified & & 48.00 \\
& High & 12 & 20.00 \\
& Intermediate & 5 & 32.00 \\
\hline
\end{tabular}

TABLE III

FREQUENCY OF EXPRESSION OF LATENT MEMBRANE PROTEIN (LMP-1) OF EBV IN HODGKIN'S LYMPHOMA (HL) AND NON-HODGKIN'S LYMPHOMA (NHL) PATIENTS ACCORDING TO GENDER AND AGE AT DIAGNOSIS.

\begin{tabular}{|c|c|c|c|c|c|}
\hline \multirow[t]{3}{*}{ Parameters } & & \multicolumn{4}{|c|}{$\begin{array}{l}\text { Result of EBV detection Positive } \\
\text { Type of lymphoma }\end{array}$} \\
\hline & & \multicolumn{2}{|c|}{$\mathrm{HL}$} & \multirow{2}{*}{\multicolumn{2}{|c|}{ NHL }} \\
\hline & & $\mathrm{N}$ & $\%$ & & \\
\hline \multicolumn{6}{|l|}{ Age groups } \\
\hline \multirow{4}{*}{ Gender } & $<50 \mathrm{yr} \mathrm{s}$ & & & & \\
\hline & & $3 / 6$ & 50.00 & $6 / 13$ & 46.15 \\
\hline & Male & & & & \\
\hline & & $12 / 20$ & 60.00 & $3 / 12$ & 25.00 \\
\hline
\end{tabular}

TABLE IV

FREQUENCY OF EXPRESSION OF LATENT MEMBRANE PROTEIN (LMP-1) OF EBV IN HODGKIN'S LYMPHOMA (HL) AND NON-HODGKIN'S LYMPHOMA (NHL) PATIENTS ACCORDING TO SUBTYPE AND GRADE.

\begin{tabular}{c|lcc}
\hline \multirow{3}{*}{ Type of lymphoma } & \multicolumn{2}{|c}{ PATIENTS ACCORDING TO SUBTYPE AND GRADE. } \\
& Subtype & $\mathrm{N}$ & $\%$ \\
\hline \multirow{5}{*}{$\mathrm{HL}$} & & & \\
& & $11 / 18$ & 61.11 \\
& Mixed Cellularity & $2 / 7$ & 28.57 \\
& Nodular Sclerosis & $2 / 2$ & 100.00 \\
& Lymphocyte Depletion & $1 / 1$ & 100.00 \\
& Lymphocyte Rich & $2 / 2$ & 100.00 \\
& Unclassified & & 25.00 \\
& High & $3 / 12$ & 60.00 \\
& Intermediate & $3 / 5$ & 25.00 \\
\hline
\end{tabular}




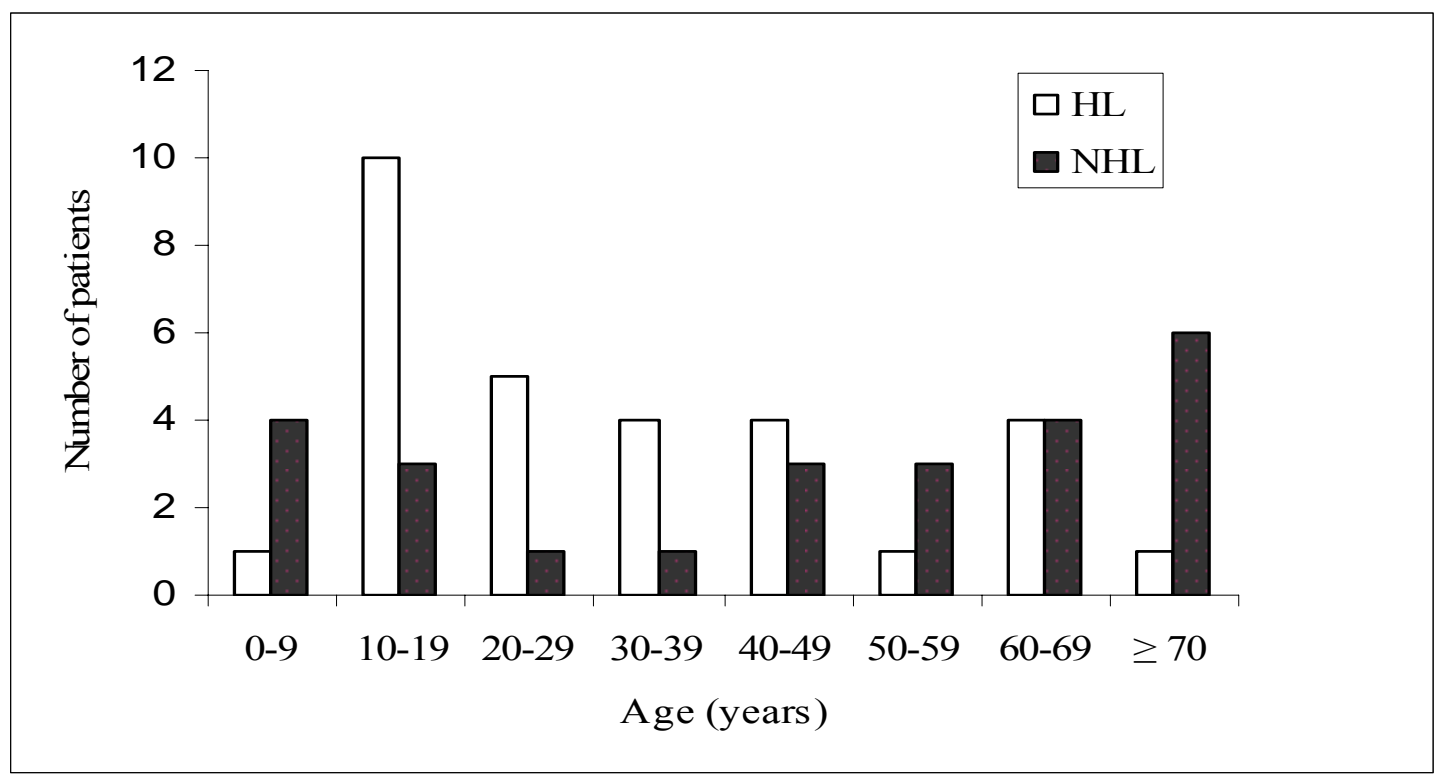

Fig. 1 Distribution of Hodgkin's lymphoma and non-Hodgkin lymphoma patients according to age
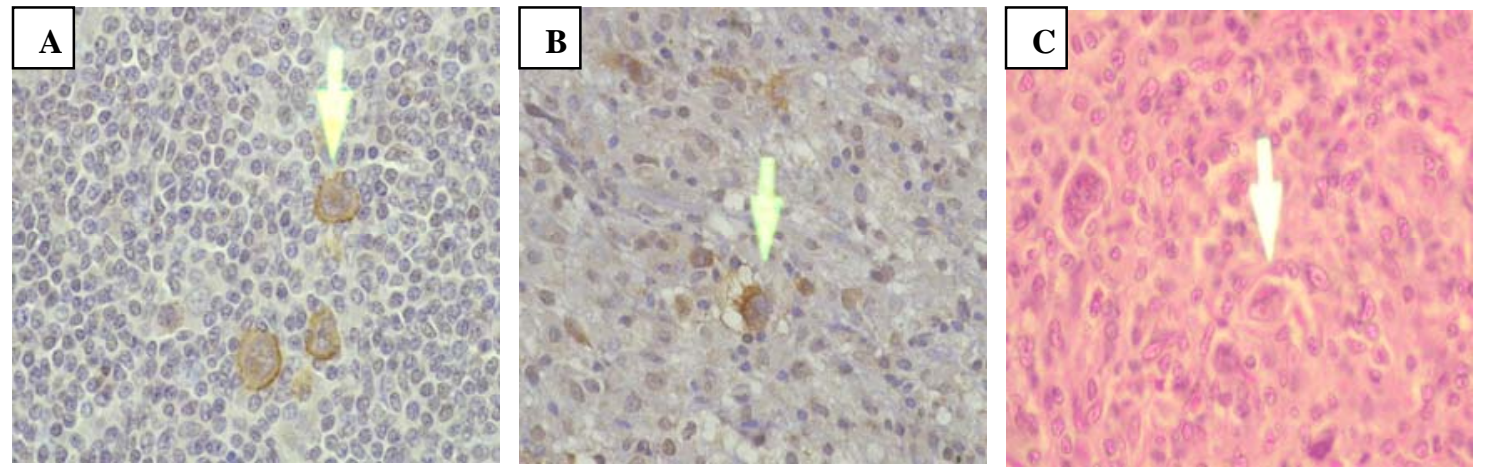

Fig. 2 Expression of latent membrane protein-1 of EBV in Hodgkin's lymphoma (HL). A. HL patient with mixed cellularity. B. HL patient with nodular sclerosis. C. HL patient with mixed cellularity (Negative control). For all slides, magnifications were 40X. Arrows inside the figure indicates the position of RS cell.
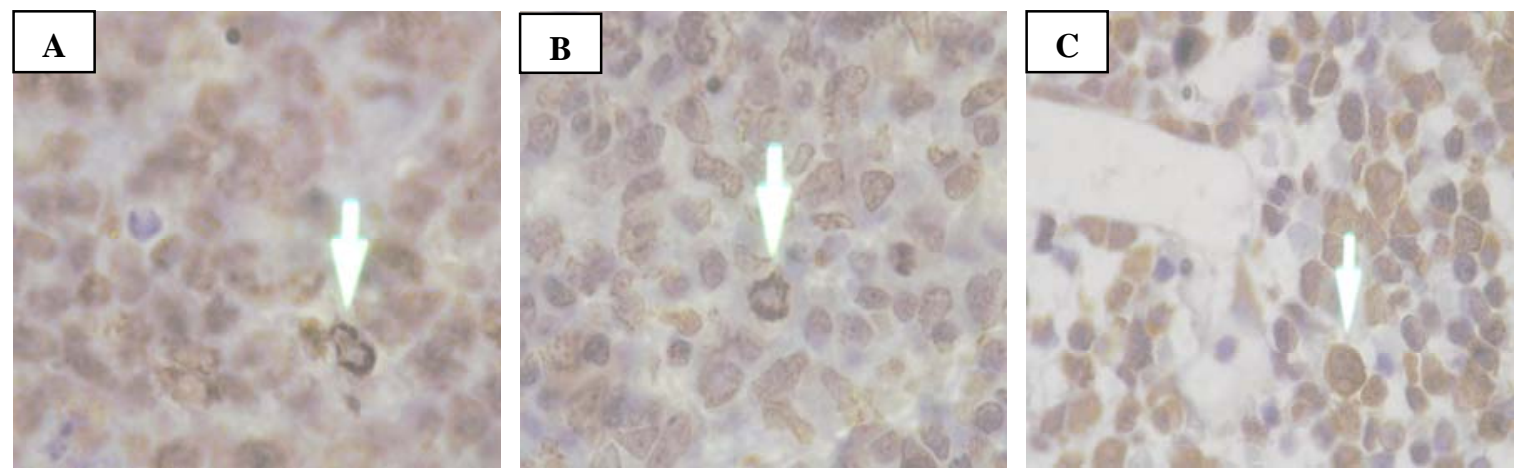

Fig. 3 Expression of latent membrane protein-1 of EBV in non-Hodgkin's lymphoma (NHL) patients. A. NHL patient with low grade. B. NHL patient with intermediate grade. C. NHL patient with high grade. For all slides, magnifications were 100X. Arrows indicate the tumor cell. 